\title{
ASSESSMENT OF QUALITY AND RISKS EXPOSURE IN HISTOPATHOLOGY DEPARTMENT
}

\section{Deacu Mariana 1,2, Așchie Mariana,12, Bălțătescu Gabriela Izabela1,2, Boșoteanu Mădălinaa,2}

${ }^{1}$ Clinical Service of Pathology, Emergency County Hospital "Sf. Apostol Andrei” Constanța

${ }^{2}$ Faculty of Medicine, University "Ovidius" of Constanța

Gabriela Izabela Bălțătescu

Clinical Service of Pathology, Emergency County Hospital, 145 Tomis Avenue, 900591 Constanta, Romania email: gabrielabaltatescu@yahoo.com phone: +40241503289

\begin{abstract}
The role of histopathology department in establishing a correct and final diagnosis is essential, this leading to an effective treatment for the patient so its healing to be complete. In order to achieve this goal, it is necessary that all complex steps belonging to this department to be properly carried out because any mistakes in a pathology report may adversely affect the patient outcome. Therefore, it is mandatory the quality assessment of all procedures from the laboratory to be constantly done so that the report to be reliable and accuracy. All three main categories of activities specific to a pathological laboratory (pre-analytic, analytic and post-analytic phases) must undergo quality control. During the progress of these phases, it also must be identified the risks and safety problems regarding the personal staff. These may be different for each activity or procedure which is performed by the laboratory workers. All the physical, chemical or biological hazards must be identified to assess, appreciate the possible risks and to implement preventive measures. The aim of this study is to highlight the importance of quality management for all phases included in a surgical pathological laboratory based on the pathological errors observed, identify emerging risks exposure and necessary measures to prevent them.
\end{abstract}

Keywords: Quality control, pathological errors, risks exposure, safety

\section{Introduction}

The anatomical pathology provides not only histopathological tests, which represent the "gold standard" for a surgical specimen analysis, but cytopathology and autopsy test too and, in the last decades, it was also enriched by important procedures such as immunohistochemistry, molecular techniques and morphometry. All of them has one purpose: to deliver an accuracy, reliable and timely pathological report, so that the patient to receive a correct and effective treatment. Thus, there are many complex steps which must be followed to obtain a useful and good pathological report. Any error along the way can be devastating for patient and can have lethal consequences. So, a lot of efforts must be done to ensure that the chance of a medical mistake is reduced to zero. The only method that can achieve this goal is to assess the quality of a pathology service, to identify the possible medical errors and, in the same time, to ensure a safe environment by evaluating the risks exposure and implementing specific preventing measurements.

Therefore, it is mandatory that the quality assessment of all procedures from the laboratory to be constantly done, so the report to be reliable and accuracy. It was cleared that quality system are required and for this reason International 
Organization for Standardization (IOS) released their guidelines - ISO 9000 to improve medical reports (1). Even if the "quality" term has several definitions, however the best one is that it represents an accurate, complete and timely report (2). The quality management, which include both quality assurance and improvement, proved to be a difficult task in anatomical pathology since its final results are more qualitative than quantitative and there are not easy methods to measure it (3).

All activities within a pathological laboratory, which form a complete cycle for test, can be broken in three main parts (pre-analytic, analytic and post-analytic examination) and all of them must undergo quality control $(4,5)$.

\section{The pre-analytic phase}

The route of a surgical specimen, from initial point of collection to the end in the pathology laboratory, is not without risk of making mistakes. All of these errors are part of the pre-analytic phase and in this category are also included the following steps: reception of the surgical probe, gross evaluation and processing the material (Tabel 1) (5). According to some studies, the pathological errors during this phase have the highest frequency, ranging from $53.3 \%$ (6) to $92.9 \%$ (7). The last one it was identified in the research of Rao S.et al. (2016) in which, from a total of 18626 tissue specimens, different errors were identified in 113 cases and most of them belonged to pre-analytic phase (7).

In the research of Wiwanitkis et al. (2001), inadequate quality of specimen is the most frequent type of error, having high rate up to $47.0 \%$ (8). Wrong identification of the patient probe was on the second place, with a frequency of $26.8 \%$, and inadequate quantity of specimen was observed in $11.6 \%$ of cases (8). The most frequent cause of wrong labelling was due to illegible handwriting or human error and these errors can be prevented by double check of the label or highlighted it (7).

Other studies have been focused on the importance of presence or absence of clinical information. The study of Nakhleh et al. (1999) emphasized the importance of having access to reliable clinical and imagistic information, 2.4\% of the cases examined didn't have clinical data
(9). In $0.73 \%$ of cases, the pathologist needed supplementary clinical information in order to finalize the pathological report and as a result, it was a delay in delivering it (9). It was also observed that the final report was changed in $6.1 \%$ of cases, consecutively knowledge of clinical or imagistic information (9)

Grossing of surgical specimens represent an important part of this phase and because comprised many different steps, there are also possible multiple types of errors. There were recorded errors like improper cut section in the block, cross-contamination with other types of tissue, defective was impregnation, technical problems within automatic processor, cryostat or microwave, power failure with no other alternative supply, poor processing due to a poor dehydration, mixing up the tissue, conducting special stains on wrong sections (7). To obtain a good quality control in this complex phase it was recommended to have a checklist in which all the preventive measure to be written (10).

\section{The analytical phase}

The analytical phase is reserved to microscopic evaluation of the slide and conceive the pathological report. Mistakes within this phase are encountered with a wide range of frequencies from $4 \%$ (11) to $42.1 \%$ (6).

The most frequent type of error is related to interpretation with serious consequences on both patient and pathologist $(10,12)$. The correctness of the final pathological report is closely related and depended by effectiveness of all pre-analytical steps: gross evaluation of the surgical specimen and sectioning of which requires special attention to sample the most representative fragments, embedding, sectioning and staining with either usual haematoxilineosin or special stains and immunohistochemical evaluation (2). It was noticed that the incidence of these types of errors were higher in the institutions with pathology residency programs or when cases were reviewed after release than in those institution in which the result was written after a second opinion (13). False negative results for cancer was one of the major error with a $63 \%$ rate in the study of Troxel et al (2005) (14). Other types of defective interpretation are represented 
by false positive results, miss-classification which means not reporting primary diagnostic characteristics (eg. positive/ negative or benign/ malignant) or secondary features (margin, grade, stage, vascular or perineural invasion) (15). The better way to avoid this type of mistake is to use one of peer review methods, which have proved to be an important measure of quality (2). There are a lot of peer-reviews methods but the most relevant are following: reviewing the results and reports within the department before releasing to other institutions, studying the cases from a specific type of disease with internal consultation, correlation between cytology and histological report or between frozen section and permanent section, intradepartmental conferences (2). Reviewing the difficult cases by a pathologist with a wide experience in a particular field of pathology, it will significantly reduce the rate of error that may occur in interpretation. A change of diagnosis after a second opinion was observed in $17 \%$ cases of breast biopsies in a study on 430 cases, most of them being observed in those cases with a benign initial diagnosis (16).

Another common error is represented by a delay of releasing the result which it can be considered equally mistake of analytical phase (6) as well as mistake of post-analytical phase (11). Turn-around time (TAT) is considered to be an extremely useful measure for this type of mistakes. It acts as a quality measure and it is defined as the time passing from receiving the surgical specimen in pathology laboratory and releasing the final report (17). It depends on surgical specimen type (its volume or complexity), number of blocks and slides examined, re-grossing (eg. for additional lymph node evaluation), recuts, re-embedding, additional techniques (overnight fixation, decalcification, special stain, immunostains, molecular test) or collaboration with other doctors $(17,18)$. A final report of at least $90 \%$ of all probes must be released in 2-3 days according to CAP suggestions (19). It was also advised that each pathological laboratory must established its routine probe (19). In 2015, Volmar et al. proved in its study which included 56 institutions and evaluated 2763 large surgical specimen that TAT of larger or complex probes is longer compared with TAT of small biopsies (18). A prolonged time was also observed for those cases with frozen sections, for specimen which required overnight fixation or special stains and for malignant cases (18). This was the reason for which it was recommended that TAT should be more than 2 days in those pathological laboratories where cancer surgical specimens are evaluated (17).

\section{The post-analytical phase}

The post-analytical phase begins with typing the histopathological report and ends with its release to the patient (Table 1). Errors falling in this category are less frequent then those observed in the previous phases, with a rate up to $8 \%$ (11). In this phase it can be encountered errors regarding the final report with wrong validation of data, a delay in TAT, improper data entry. Defective reports refer both to reports with wrong or missing information not related to pathological report, as clinician's name or date of procedure and those reports with different typing errors (20). It can comprise mistakes regarding the modality of reporting the results and interpretation in the settings of clinical background, errors regarding the releasing the final diagnosis to the correct patient or inadequate storage conditions $(5,20)$.

\section{Pathological errors}

The rate of pathology report errors represents an indicator of quality. They can be classified both in terms of way of occurrence and in terms of impact that they have on the patient. Regarding the modality of production, medical errors can be divided in four groups: errors regarding the modality of a report, errors involving the specimen, mistakes in identification and in interpretation $(13,15)$. The last category comprises the vast majority of pathological errors with a rate up to $14.6 \%$ (21), the most frequent being, in descending order, false negative-types of mistakes, false positive results and misclassification (20). Another important category of errors, with a frequency up to $13,3 \%(21)$, is represented by those related to identification of the patient or the surgical specimen, the correct anatomic location or laterality, most of them belonging to the preanalytical phase (20). 
From point of view of the impact on patient care, errors can be divided in the following categories: no impact, minimal harm, minor harm, moderate harm and major harm (15). Those errors which don't have impact on patient care are those mistakes which haven't been transmitted or have been ignored. In the category with minimal morbidity there are delay in reporting a final diagnose (no more than 6month), delay in therapy no more than 6 months or over treatment because of inappropriate diagnose. The last category includes those errors which major consequence on the quality of patient like loss of an organ or its function (15). In a study of Goldschmidt et al., severe errors were identified with a $12 \%$ frequency (22).

\section{Identification of risks exposure}

The risks at which the employees are exposed can be numerous and these are depending on different steps that are performed in the pathology laboratory, starting from the first step (receiving sample). Evaluation of the potential risks is important in order to identified and to incorporate preventive safety measures.

There are physical, chemical and biological hazards which can be identified in every anatomical pathological laboratory and for each of them there are mandatory to know and to apply all the necessary safe procedures. Ejilemele AA et al. (2005) proved in her study that employee safety is jeopardized by mitigating the risk hazards, by ignoring prevention measures and the non-recognition procedure (23).

The highest rate for physical risks obtained

Table 1. Different types of errors specific for all three phases of a complete cycle test which affect the final pathological report.

Pre-analytical phase $(7,8,20,21)$

Involving the surgical specimen
Accessioning (the surgical specimen isn't the same as in request; losing the probe; wrong number for labeling the probe; incorrect attachment of worksheets to the gross description)

Inaccurate quality or quantity of specimen

Use an unsuitable container and/or mislabeling it

Inadequate fixation (wrong volume/poor quality of fixation solution or insufficient/ prolonged time for fixation)

Errors in macroscopic evaluation regarding the correct orientation, localization, laterality, measurements or proper samples.

Mistakes during tissue processing due to technical problems (power failure, incorrect was impregnation, different technical fault of automatic tissue processor or of cryostat, poor dehydration)

Involving the patient Wrong identification data of the patient because of wrong labelling Mixing up the surgical probes

Not having access to clinical data regarding the history of the patient or to different initial laboratory tests.

\begin{tabular}{ll}
\hline Analytical phase $(2,6,11,12)$ & \\
\hline Intra-operative & Differences between diagnosis of frozen-section and permanent section \\
\hline Final diagnosis & Slides with technical defects \\
& Foreign tissue on the slide \\
& Incorrect block/ slide identification \\
& Interpretation errors \\
& A prolonged time for a final diagnose. \\
& Errors regarding the immunohistochemistry (using expired antibodies; absence of \\
& external validation for different biomarkers) . \\
\hline Post-analytic phase $(11,12)$ & \\
\hline & Errors in transcription of the final pathological report \\
& Defective reports \\
& Delay in releasing the diagnosis. \\
& Inadequate storage conditions. \\
\hline
\end{tabular}


in the study of Howard Huges Medical Institute Office of Laboratory Safety were laceration lesion (32\%), followed by bruise, sprain, strain, fracture $(21 \%)$, chemical exposure (11\%) and eye injury (10\%) (24). A possible source of injuries is represented by the electrical laboratory equipment which can be dangerous as there is an increased risk for electrical shock, but it can be prevented by proper installation and grounding. Sharps objects or broken glass must be quickly and with great care removed in special container and after this it must be seal. Laboratory glassware and plasticware must be put in cardboard boxes while there are transporting through the laboratory and if they are contaminated, they must be de-contaminated. As a rule of prevention, the boxes are not used for the disposal of liquid wastes, biohazardous materials or chemically contaminated objects $(25,26)$.

Regarding chemical hazards, the most common routes for endanger the safe of the staff are inhalation, absorption through the skin or, rarely, by ingestion. The most frequent chemical substances are toluene, formaldehyde and xylene. To prevent any risk of exposure, all of these toxic chemicals must be handled in their containers, labelled with their usual names and concentration, stored in special area which are well-ventilated $(26,27)$.

Biological hazards represent the most established and recorded cause for injuries related to a laboratory (28). They represent a frequent source of infections and in a study conducted through a period of 20 years in the United State of America it was showing that Mycobacterium tuberculosis infection was the most frequent bacteria, with $24.5 \%$ rate from all the biological agents identified (29). Aerosols are responsible for most of the infections for this reason the laboratories must have a continuous airflow and filtration of exhausted air

Safety in a surgical pathology laboratory must be a priority for all managers. It can be improved when there is a safety manual with instructions and procedures, which is regular update and continuous improved. A key role in achieving a safe environment for both employees and patients are safety training courses which must be evaluated and constantly implemented. All employees must be trained and informed about the potential risks (28). Safety is not a passive process, instead is requiring for continuous review. When it is neglected, it can increase the risk for deteriorate de health of staff and it can destroy the equipment and facilities (25). It is closely related to quality control and there are necessary to do regular audit to ensure a safe environment $(26,28)$.

\section{Enclusion}

Identification of specific pathological errors in an anatomic laboratory for each phase of the entire process circle and applying tailored preventing measures can lead to the improved of the quality of the results. The most important and significant quality indicators are accuracy and complete of the final diagnose along with timely pathological report. Therefore, it is important to implement quality system and to improve them all the time. Last but not least, the enforcement of safety and accident prevention in the laboratory provide a poper environment for all lab activities and provide a good protection for both patient and staff.

\section{References}

1. Schlickman JJ. ISO 9000 quality management system design: optimal design rules for documentation, implementation, and system effectiveness: ASQ Quality Press; 1998.

2. Nakhleh R. What is quality in surgical pathology? Journal of clinical pathology. 2006;59(7):669-72.

3. Iyengar JN. Quality control in the histopathology laboratory: an overview with stress on the need for a structured national external quality assessment scheme. Indian $\mathrm{J}$ Pathol Microbiol. 2009;52(1):1-5.

4. Hollensead SC, Lockwood WB, Elin RJ. Errors in pathology and laboratory medicine: consequences and prevention. J Surg Oncol. 2004;88(3):161-81.

5. Nakhleh RE. Patient safety and error reduction in surgical pathology. Arch Pathol Lab Med. 2008;132(2):181-5.

6. TosunerZ, GucinZ, Kiran T, Buyukpinarbasili N, Turna S, Taskiran O, et al. A Six Sigma Trial For Reduction of Error Rates in 
Pathology Laboratory. Turk Patoloji Derg. 2016;32(3):171-7.

7. Rao S, Masilamani S, Sundaram S, Duvuru P, Swaminathan R. Quality Measures in Pre-Analytical Phase of Tissue Processing: Understanding Its Value in Histopathology. J Clin Diagn Res. 2016;10(1):EC07-11.

8. Wiwanitkit V. Types and frequency of preanalytical mistakes in the first Thai ISO 9002: 1994 certified clinical laboratory, a 6month monitoring. BMC Clinical Pathology. 2001;1(1):5.

9. NakhlehRE, Gephardt G,Zarbo RJ. Necessity of clinical information in surgical pathology: a College of American Pathologists Q-Probes study of 771475 surgical pathology cases from 341 institutions. Archives of Pathology and Laboratory Medicine. 1999;123(7):6159.

10. Adyanthaya S, Jose M. Quality and safety aspects in histopathology laboratory. J Oral Maxillofac Pathol. 2013;17(3):402-7.

11. Samulski TD, Montone K, LiVolsi V, Patel K, Baloch Z. Patient Safety Curriculum for Anatomic Pathology Trainees: Recommendations Based on Institutional Experience. Adv Anat Pathol. 2016;23(2):112-7.

12. Santana MF, Ferreira LCL. Diagnostic errors in surgical pathology. Jornal Brasileiro de Patologia e Medicina Laboratorial. 2017;53(2):124-9.

13. Meier FA, Varney RC, Zarbo RJ. Study of amended reports to evaluate and improve surgical pathology processes. Adv Anat Pathol. 2011;18(5):406-13.

14. Troxel DB. An insurer's perspective on error and loss in pathology. Archives of Pathology and Laboratory Medicine. 2005;129(10):1234-6.

15. Zarbo RJ, Meier FA, Raab SS. Error detection in anatomic pathology. Arch Pathol Lab Med. 2005;129(10):1237-45.

16. Romanoff AM, Cohen A, Schmidt H, Weltz CR, Jaffer SM, Nagi CS, et al. Breast pathology review: does it make a difference? Annals of surgical oncology. 2014;21(11):3504-8.

17. Patel S, Smith JB, Kurbatova E, Guarner J. Factors that impact turnaround time of surgical pathology specimens in an academic institution. Human pathology. 2012;43(9):1501-5.

18. Volmar KE, Idowu MO, Souers RJ, Karcher DS, Nakhleh RE. Turnaround time for large or complex specimens in surgical pathology: a College of American Pathologists Q-Probes study of 56 institutions. Arch Pathol Lab Med. 2015;139(2):171-7.

19. Novis DA, Zarbo RJ, Saladino AJ. Interinstitutional comparison of surgical biopsy diagnosis turnaround time: a College of American Pathologists Q-Probes study of 5384 surgical biopsies in 157 small hospitals. Arch Pathol Lab Med. 1998;122(11):951-6.

20. Karki S. Errors: Detection and minimization in histopathology laboratories. Journal of Pathology of Nepal. 2015;5(10):859-64.

21. Volmar KE, Idowu MO, Hunt JL, Souers RJ, Meier FA, Nakhleh RE. Surgical pathology report defects: a College of American Pathologists Q-Probes study of 73 institutions. Arch Pathol Lab Med. 2014;138(5):602-12.

22. Goldschmidt H, Lent R. Gross errors and work flow analysis in the clinical laboratory. Klin Biochem Metab. 1995;3(131):40.

23. Ejilemele A, Ojulu A. Knowledge, attitude and practice of aspects of laboratory safety in Pathology Laboratories at the University of Port Harcourt Teaching Hospital, Nigeria. Nigerian Journal of clinical practice. 2005;8(2):102-6.

24. Howard Hudges Medical Institute. Lab Safety 2017 [Available from: http://www. hhmi.org/research/labsafe/index.html.

25. World Health Organization. Laboratory quality management system: handbook 2005 [Available from: http://www.who.int/ihr/ publications/lqms/en/.

26. International Organization for Standardization. ISO 15190:2003. Clinical Laboratory Medicine - Safety in Medical Laboratories 2009 [Available from: https:// www.iso.org/standard/38477.html.

27. Dapson JC, Dapson RW. Hazardous materials in the histopathology laboratory: regulations, risks, handling and disposal: Anatech Limited; 2005.

28. Noble MA. ISO 15190: 2003 MEDICAL 
LABORATORIES-REQUIREMENTS FOR

SAFETY [Available from: http://www.ifcc. org/ejifcc/vol15no4/150412200405.htm.

29. Harding AL, Brandt BK. Epidemiology of laboratory-associated infections. In: Fleming DO, Hunt DL, editors. Biological Safety: Principles and Practices: ASM Press; 2000. p. $35-54$. 